\title{
Comparación entre ligadura con lazo hemostático y engrapadora mecánica lineal para el cierre de base apendicular
}

\author{
Comparison between endoloop and linear mechanical stapler for the appendicular \\ stump closure
}

\author{
Juan J. Granados-Romero ${ }^{1}$, Alan I. Valderrama-Treviño², Baltazar Barrera-Mera ${ }^{3 *}$, Karen Uriarte-Ruíz', \\ Rodrigo Banegas-Ruiz ${ }^{5}$ y Jesús C. Ceballos-Villalva ${ }^{4}$
}

'Servicio de Cirugía General, Hospital General de México, Facultad de Medicina, UNAM; ${ }^{2}$ Laboratorio de Inmunoterapia Experimental e Ingeniería de Tejidos, Facultad de Medicina, UNAM; ${ }^{3}$ Departamento de Fisiología, Facultad de Medicina, UNAM; ${ }^{4}$ Programa de Apoyo y Fomento a la Investigación Estudiantil (AFINES), Facultad de Medicina, UNAM; ${ }^{5}$ Servicio de Cirugía de Mano y Microcirugía, Instituto Nacional de Rehabilitación. Ciudad de México, México

\begin{abstract}
Resumen
Introducción: En las últimas décadas es creciente el abordaje por mínima invasión de patologías abdominales debido a sus beneficios evidentes. El cuadro apendicular es la principal emergencia quirúrgica, con diferentes métodos del cierre de la base apendicular. En este artículo comparamos dicho cierre con engrapadora lineal o ligadura con lazo hemostático, para analizar la frecuencia de complicaciones como absceso, dehiscencia y seroma. Método: Se realizó un estudio prospectivo, observacional y descriptivo, con un total de 703 procedimientos, empleando en 567 pacientes ligadura con lazo hemostático y en 136 engrapadora lineal, operados por los mismos cirujano y equipo quirúrgico, con curva de aprendizaje concluida. Resultados: Las complicaciones referidas en el presente estudio son absceso $(n=5)$, dehiscencia $(n=3)$ y seroma $(n=3)$. De acuerdo con las fases de la patología apendicular: fase 10 apéndice congestivo, no presentaron complicaciones; fase 2 o supurativo, se reportó un caso de dehiscencia de herida quirúrgica con el uso de ligadura con lazo hemostático; fase 3 o necrótico, se reportó un caso de seroma en un paciente tratado con ligadura con lazo hemostático; y fase 4 o perforado, se encuentra diferencia significativa en el caso de abscesos, reportando cinco con el uso de ligadura con lazo hemostático y ninguno con engrapadora lineal. Conclusiones: En nuestro estudio no existe diferencia estadísticamente significativa entre el uso de engrapadora lineal o ligadura con lazo hemostático en las fases apendiculares 1-3; en la fase 4 es de utilidad significativa el uso de engrapadora lineal ante la incidencia de abscesos.
\end{abstract}

PALABRAS CLAVE: Ligadura con lazo hemostático. Engrapadora mecánica lineal. Apendicitis. Base apendicular.

\begin{abstract}
Introduction: In the last decades, the approach by minimally invasive surgery of abdominal pathologies is growing due to its evident benefits; the appendicular cases being the main surgical emergency, with different methods of closing the appendicular base. In this article, we compared the appendicular base closure with linear stapler and endoloop, to analyze the frequency of complications such as abscess, dehiscence and seroma. Method: A prospective, observational and descriptive study was conducted, with a total of 703 procedures, using 567 endoloop patients and 136 linear stapler, operated by the same
\end{abstract}

\author{
Correspondencia: \\ *Baltazar Barrera-Mera \\ Departamento de Fisiología \\ Facultad de Medicina, UNAM \\ Av. Universidad 3000 \\ Del. Coyoacán \\ Fecha de recepción: 14-03-2018 \\ C.P. 04510, Ciudad de México, México \\ Fecha de aceptación: 15-05-2018 \\ E-mail: baltazar.barrera.mera@gmail.com \\ DOI: $10.24875 / C I R U .18000258$ \\ Cir Cir. 2018;86:428-431 \\ Contents available at PubMed \\ www.cirugiaycirujanos.com
}


surgeon and surgical team, with a completed learning curve. Results: The complications referred in the present study were patients with abscess $(n=5)$, dehiscence $(n=3)$ and seroma $(n=3)$. According to the phases of the appendiceal pathology: phase 1 or congestive appendix did not present complications; phase 2 or suppurative was reported one case of surgical wound dehiscence in the use of endoloop; in phase 3 or necrotic, one case of seroma was reported in a patient treated with endoloop; while in phase 4 or perforated there is a significant difference in the case of abscesses, reporting five in the use of endoloop and none in the case of a linear stapler. Conclusions: In our study there is no statistically significant difference between the use of linear stapler or endoloop in the early appendicular phases; being of significant utility in Phase 4 the use of linear stapler for the incidence of abscesses.

KEY WORDS: Endoloop. Mechanical lineal stapler. Appendicitis. Appendicular stump.

\section{Introducción}

Durante las últimas décadas ha sido creciente el abordaje por mínima invasión de patologías abdominales, especialmente en cuadros agudos ginecológicos, vesiculares y apendiculares, en los que se ha logrado ofrecer una pronta recuperación, disminución del dolor posoperatorio y una baja frecuencia de infecciones del sitio quirúrgico, comparado con la cirugía abierta tradicional ${ }^{1-4}$.

El cuadro apendicular agudo es la emergencia quirúrgica abdominal más frecuente, cuyo abordaje actual de resolución es preferente mediante apendicectomía laparoscópica, con diferentes métodos para el cierre de la base apendicular, de los cuales los más utilizados son la ligadura con lazo hemostático, las suturas, los endoclips y las engrapadoras lineales. Un cierre ideal debe ser resistente a la tracción, evitar infecciones o contaminación, y presentar una reacción local mínima en relación con el tejido en que es colocado ${ }^{5}$. En este artículo comparamos dos métodos de cierre de base apendicular, engrapadora lineal y ligadura con lazo hemostático, con el objetivo de determinar si existe diferencia entre la frecuencia de complicaciones como absceso, dehiscencia y seroma. La ligadura con lazo hemostático suele ser más económica que la engrapadora lineal, la cual además requiere una incisión de trocar que puede requerir desde 12 hasta $15 \mathrm{~mm}$, con el beneficio de permitir un cierre rápido y eficaz de la base apendicular, incluso en situaciones de perforación apendicular-9.9. En relación con la duración de una cirugía laparoscópica utilizando engrapadora lineal, esta es de 50 a 62 minutos, mientras que el tiempo reportado con el uso de la ligadura con lazo hemostático es de 47 a 75.4 minutos $^{10}$.

\section{Método}

Se realizó un estudio prospectivo, observacional y descriptivo, con 703 pacientes sometidos a apendicetomías durante el periodo de enero 2014 a diciembre 2017, por vía laparoscópica, en diferentes hospitales privados, operados por el mismo cirujano con curva de aprendizaje superada. Este protocolo fue aprobado por el comité de ética e investigación del Hospital Ángeles Clínica Londres, y a cada paciente se le solicitó autorización mediante un consentimiento informado, en el que podía aceptar o rechazar su ingreso en el estudio. En total se incluyeron 703 procedimientos, de los cuales en 567 se utilizó ligadura con lazo hemostático y en 136 engrapadora lineal, realizados por los mismos cirujano y equipo quirúrgico, con curva de aprendizaje concluida. La base informática de los expedientes electrónicos se recolectó en Microsoft Excel 2016, y para el análisis estadístico se utilizó la prueba exacta de Fisher en el programa $\mathrm{R}$, tomando como significancia estadística un valor de $p<0.05$.

\section{Resultados}

Se incluyeron 703 pacientes que fueron intervenidos de apendicectomía laparoscópica; en 567 se utilizó pare el cierre de base apendicular la ligadura con lazo hemostático (80.65\%) y en $136(19.34 \%)$ se utilizó engrapadora mecánica.

Se estudió y analizó la incidencia de complicaciones: absceso $(n=5)$, dehiscencia $(n=3)$ y seroma $(n=3)$. Los pacientes se agruparon según la fase del cuadro apendicular (fases 1 a 4). La fase 1 corresponde a un apéndice congestivo; la fase 2 , supurativo; la fase 3, necrótico; y la fase 4, perforado. Una vez dividido por fases, se registró el número de pacientes que fueron intervenidos con ligadura con lazo hemostático o con engrapadora para compararlos y determinar si existe diferencia significativa en la incidencia de complicaciones.

Dentro de la fase 1 apendicular no se encontró ninguna de las complicaciones previamente mencionadas. En la fase 2 se encontró una dehiscencia. En 
la fase 3 hubo un caso de seroma con el uso de ligadura con lazo hemostático, pero no existe diferencia estadísticamente significativa. En la fase 4 se encuentra diferencia significativa para la incidencia de abscesos, ya que ocurrieron cinco abscesos al usar ligadura con lazo hemostático y ninguno al usar engrapadora lineal; para las complicaciones restantes no existe diferencia significativa entre el uso de las dos técnicas quirúrgicas (Tabla 1).

\section{Discusión}

En los 703 pacientes que se sometieron a este procedimiento, solo hubo 11 complicaciones (1.56\%), de las cuales $10(1.42 \%)$ fueron con el uso de ligadura con lazo hemostático y $1(0.14 \%)$ correspondió a la engrapadora lineal. Dentro de dichas complicaciones hubo 5 abscesos $(0.71 \%), 3$ dehiscencias $(0.42 \%)$ y 3 seromas $(0.42 \%)$. De acuerdo con Hilko Swank et al. ${ }^{4}$, la prevalencia de abscesos intraabdmoniales después de la apendicetomía laparoscópica es del $4.2 \%$, y la de infecciones de heridas es del $0.5 \%$.

Al realizar el análisis estadístico encontramos lo siguiente: en las fases 1,2 y 3 , el valor de $p$ fue $<0.05$, por lo cual no podemos concluir que un método de cierre sea mejor que el otro; en la fase 4 ocurre lo mismo en cuanto a las complicaciones como seroma y absceso, pero para la dehiscencia observamos un valor de $p<0.05$, por lo cual podemos concluir que, en esta fase, la engrapadora presenta menos abscesos que la ligadura con lazo hemostático.

La cirugía laparoscópica ha mostrado tener numerosos beneficios para la resolución de diversas patologías, como la apendicitis, pero persiste la controversia sobre el método de cierre de la base apendicular.

Van Rossem, et al. ${ }^{11}$, en el año 2017, realizaron una comparación entre ligadura con lazo hemostático y engrapadora para la resolución de apendicitis por vía laparoscópica con 1369 pacientes, con el objetivo de analizar una posible relación entre el método de cierre y las complicaciones infecciosas, y concluyeron que el tipo de cierre no influía en las complicaciones, pero sí la gravedad de la apendicitis. Sahm, et al. ${ }^{12}$ evaluaron la efectividad del uso de ligadura con lazo hemostático para el cierre apendicular en comparación con la engrapadora lineal, y hallaron que la ligadura con lazo hemostático no tiene diferencia significativa en el resultado de complicaciones. Miyano, et al..$^{13}$ realizaron un estudio similar y concluyeron que no hubo diferencia significativa en las complicaciones de la
Tabla 1. Comparación de la incidencia de complicaciones en el cierre de la base apendicular

\begin{tabular}{llccc}
\hline Fase & Complicación & $\begin{array}{c}\text { Ligadura con lazo } \\
\text { hemostático }\end{array}$ & Engrapadora & $\mathbf{p}$ \\
\hline $1 \quad$ Absceso & 0 & 0 & $>0.05$ \\
& Dehiscencia & 0 & 0 & $>0.05$ \\
2 & Absceso & 0 & 0 & $>0.05$ \\
& Dehiscencia & 1 & 0 & $>0.05$ \\
3 & Absceso & 0 & 0 & $>0.05$ \\
& Dehiscencia & 0 & 0 & $>0.05$ \\
& Seroma & 1 & 0 & $>0.05$ \\
4 & Absceso & 5 & 0 & 0.00178 \\
& Dehiscencia & 1 & 1 & 0.0518 \\
& Seroma & 2 & 0 & 0.08688 \\
\hline
\end{tabular}

apendicetomía laparoscópica entre el uso de engrapadora o de ligadura con lazo hemostático. Safavi, et al..$^{14}$ observaron que el uso de ligadura con lazo hemostático es preferible, ya que no existe diferencia significativa con la engrapadora en cuanto a complicaciones, pero sí en relación a costo-beneficio, y así mismo declararon que dichas complicaciones pueden deberse a la calidad del muñón apendicular en lugar de a la técnica de cierre. Por el contrario, Escolino, et $a .^{15}$ concluyeron que, a pesar de que la engrapadora es más costosa que la ligadura con lazo hemostático, esta debe usarse en apendicitis complicadas con perforación o necrosis, ya que se asoció con una menor incidencia de abscesos.

En cuanto a costo-beneficio, Beldi, et al. ${ }^{16,17}$ demostraron que, en Suiza, el uso de engrapadora cuesta $248 €$ más que el uso de ligadura con lazo hemostático. Kazemier, et al..$^{18}$ mencionan que, en la Unión Europea, el uso de engrapadora aumenta $300 €$ el costo en comparación con la ligadura con lazo hemostático. Miyano, et al..$^{13}$ dicen que en los EE.UU. el costo operativo promedio utilizando ligadura con lazo hemostático es de US\$890, mientras que usando engrapadora es de US\$1300. Mehdorn, et al. ${ }^{10}$ reportan que, en Alemania, el uso de engrapadora resulta $235 €$ más caro que la ligadura con lazo hemostático.

En este artículo evaluamos dos métodos, la engrapadora lineal y la ligadura con lazo hemostático. La engrapadora lineal deja grapas metálicas en el muñón y puede causar futuras adherencias en la cavidad abdominal o la formación de pseudopólipos en el ciego. En cuanto a la ligadura con lazo hemostático, hay evidencia de que reduce su seguridad al usarla en un apéndice perforado ${ }^{4}$. Una parte crucial del procedimiento con este método es la fijación manual de la 
ligadura, con posibilidad de cierre insuficiente y peligro de un absceso del muñón apendicular ${ }^{5}$. La ligadura con lazo hemostático es de 6 a 12 veces más económica que la engrapadora lineal ${ }^{6}$.

\section{Conclusiones}

En nuestro estudio no existe diferencia estadísticamente significativa entre el uso de engrapadora lineal o ligadura con lazo hemostático en las fases apendiculares 1,2 y 3 . No se observa el mismo comportamiento en la fase 4, en particular para el absceso, que presenta mayor incidencia con el uso de ligadura con lazo hemostático. Debido a esto, consideramos que podría ser adecuado y oportuno el uso de engrapadora lineal para el cierre de la base apendicular en la fase 4.

\section{Conflicto de intereses}

Los autores declaran no tener ningún conflicto de intereses.

\section{Bibliografía}

1. Sauerland S, Jaschinski T, Neugebeauer EA. Laparoscopic versus open surgery for suspected appendicitis. Cochrane Databases Syst Rev. 2010;(10):CD001546.

2. Starvos A, Dimitrios M, Shahab H, Hajibandeh S, Antoniou G, Gorter R, et al. Optimal stump management in laparoscopic appendectomy: a network meta-analysis by the minimally invasive surgery synthesis of interventions and outcomes network. Surgery. 2017;162:994-1005.

3. Taguchi Y, Komatsu. S, Sakamoto E, Norimizu D, Shingu Y, Hasegawa. Laparoscopic versus open surgery for complicated appendicitis in adults: a randomized controlled trial. Surg Endosc. 2016;30:1705-12.
4. Swank HA, Van Rossem CC, Van Geloven AAW, In't Hof KH, Kazemier G, Meijerink WJHJ, et al. Endostapler or endoloops for securing the appendiceal stump in laparoscopic appendectomy: a retrospective cohort study. Surg Endosc. 2014;28:576-83.

5. Delibegović S, Iljazović E, Katica M, Koluh A. Tissue reaction to absorbable endoloop, nonabsorbable titanium staples, and polymer Hem-o-lok clip after laparoscopic appendectomy. JSLS. 2011;15:70-6.

6. Naiditch J, Lautz T, Chin A, Browne M, Rowell E. Endoloop as the first line tool for appendiceal stump closure in children with appendicitis. Eur J Pediatr Surg. 2015;25:155-9.

7. Rakić M, Jukić M, Pogorelić Z, Mrklić I, Kliček R, Družijanić N, et al. Analysis of endoloops and endostaples for closing the appendiceal stump during laparoscopic appendectomy. Surg Today. 2014;44:1716-22.

8. Bozkurt MA, Unsal MG, Kapan S, Gonenc M, Dogan M, Kalayci MU, et al. Is laparoscopic appendectomy going to be standard procedure for acute appendicitis; a 5-year single center experience with 1,788 patients. Eur J Trauma Emerg Surg. 2014;41:87-9.

9. Al-Temimi MH, Berglin MA, Kim EG, Tessier DJ, Johna SD. Endostapler versus Hem-O-Lok clip to secure the appendiceal stump and mesoappendix during laparoscopic appendectomy. Am J Surg. 2017;214:1143-8.

10. Mehdorn M, Schurmann $\mathrm{O}$, Mehdorn MH, Gockel I. Intended cost reduction in laparoscopic appendectomy by introducing the endoloop: a single center experience. BMC Surg. 2017;17:80.

11. Van Rossem CC, van Geloven AAW, Schreinemacher MHF, Bemelman WA. Endoloops or endostapler use in laparoscopic appendectomy for acute uncomplicated and complicated appendicitis: no difference in infectious complications. Surg Endosc. 2017;31:178-84.

12. Sahm M, Kube R, Schmidt S, Ritter C, Pross M, Lippert H. Current analysis of endoloops in appendiceal stump closure. Surg Endosc. 2011;25:124-9.

13. Miyano G, Urao M, Lane G, Kato Y, Okazaki T, Yamataka A. Appendiceal stump closure in children with complicated appendicitis: a prospective analysis of endoloops versus endostaples. Asian J Endosc Surg. 2011;4:116-9.

14. Safavi A, Langer M, Skarsgard ED. Endoloop versus endostapler closure of the appendiceal stump in pediatric laparoscopic appendectomy. Can J Surg. 2012;55:37-40.

15. Escolino M, Bacmeur F, Saxena A, Holger T, Holcomb G, Esposito C. Endoloop versus endostapler: what is the best option for appendiceal stump closure in children with complicated appendicitis? Results of a multicentric international survey. Surg Endosc. 2018;32:3570-5.

16. Beldi G, Muggli K, Helbing C, Schlumpf R. Laparoscopic appendectomy using endoloops: a prospective, randomized clinical trial. Surg Endosc. 2004;18:749-50.

17. Beldi G, Vorburger SA, Bruegger LE, Kocher T, Inderbitzin D, Candinas $\mathrm{D}$. Analysis of stapling versus endoloops in appendiceal stump closure. Br J Surg. 2006;93:1390-3.

18. Kazemier G, In't Hof KH, Saad S, Bonjer HJ, Sauerland S. Securing the appendiceal stump in laparoscopic appendectomy: evidence for routine stapling? Surg Endosc. 2006;20:1473-6. 